\title{
Insectos e infestaciones asociadas al follaje de Nothofagus antarctica (Forst) Oerst (Nothofagaceae) en la cuenca del río Baker, Región de Aysén, Chile.
}

\author{
Insects and infestations associated to foliage of Nothofagus antarctica (Forst) Oerst. \\ (Nothofagaceae) in the Baker river basin, Region de Aysén, Chile.
}

\section{Olivia Vergara* \& Viviane Jerez}

Departamento de Zoología, Facultad de Ciencias Naturales y Oceanográficas, Universidad de Concepción, Casilla 160-C, Concepción, Chile.

*E-mail: olvergar@udec.cl.

\begin{abstract}
RESUMEN
En la Región de Aysén, Chile, Nothofagus antarctica (ñirre) forma bosques puros de tipo achaparrado y matorrales que corresponden a bosques mixtos secundarios de tipo arbustivo originados por acción antrópica. Este trabajo caracteriza la fauna de insectos e infestaciones asociadas al follaje de ñirre, en las coberturas vegetacionales bosque achaparrado y matorral presentes en la cuenca del río Baker. Los insectos de follaje fueron recolectados en primavera (octubre 2006) y verano (enero 2007), en cinco bosques achaparrados y 10 matorrales entre los $47^{\circ} 2^{\prime} \mathrm{S}-72^{\circ} 48^{\prime} \mathrm{W}$ y los $47^{\circ} 15^{\prime} \mathrm{S}-73^{\circ} \mathrm{W}$. Identificamos seis órdenes de insectos, de los cuales Coleoptera fue el más diverso y donde el gremio de fitófagos fue el más representativo para ambas coberturas y estaciones climáticas. No se encontró diferencias significativas en la composición taxonómica de los insectos ni en la abundancia promedio de los gremios en relación a coberturas y estaciones climáticas. Adicionalmente, se destaca la abundante defoliación causada en el ñirre por larvas de Ormiscodes sp. e infestaciones por plantas parásitas, hongos parásitos y cecidias inducidas por insectos. Muestreos más exhaustivos en las demás estaciones del año (otoño e invierno) complementados con otros métodos de muestreo, permitirían identificar la diversidad anual de especies de insectos asociadas al follaje de ñirre.
\end{abstract}

Palabras clave: bosque achaparrado, matorral, ñirre, diversidad taxonómica.

\begin{abstract}
In the Region de Aysen, Chile, Nothofagus antarctica (ñirre or Antarctic beech) forms pure forests called shrubby beech forests and secondary mixed shrub of anthropogenic origin. The aim of this work is to characterize the insect fauna associated to the foliage of Antarctic beech forests and scrublands in the Baker river basin. Insects were collected in spring (October 2006) and summer (January 2007), in five beech forests and 10 scrub patches from latitudes $47^{\circ} 2^{\prime} \mathrm{S}-72^{\circ} 48^{\prime} \mathrm{W}$ to $47^{\circ} 15^{\prime}$ $\mathrm{S}-73^{\circ} \mathrm{W}$. Six orders of insects were identified, being Coleoptera the most diverse. The phytophagous-beetle guild was the most representative in both forest types and climatic seasons. No significant differences were found in insect taxonomic composition or mean abundance of guilds between forest types or climatic seasons. Additionally, abundant defoliation by Ormiscodes sp. larvae, infestation by parasitic plants and fungi, and insect galls were observed. More exhaustive samplings in autumn and winter, complemented with more efficient sampling methods, would allow identifying the year-round insect diversity associated to Antarctic beech foliage.
\end{abstract}

KEYwords: shrubby beech forests, scrublands, Antarctic beech, taxonomic diversity.

\section{INTRODUCCIÓN}

La preferencia que el gremio de insectos herbívoros establece con sus plantas hospederas, puede ser interpretado ecológicamente como resultado de procesos de selección hacia plantas que les proporcionen una buena calidad nutricional (Centella et al. 2003), por la disposición de las ramas hacia una mayor o menor radiación solar (Rousseaux et al. 2004) o bien evolutivamente como resultado de relaciones mutualistas (Jerez 2006; Medel et al. 2002).

Nothofagus antarctica (Forst.) Oerst. (ñirre o ñire) es una 
especie caducifolia endémica del sur de Chile y Argentina, que por su tolerancia ecológica forma bosques puros y/o mixtos acompañados de otras especies leñosas. Por otra parte, es un componente importante de la dinámica colonizadora postfuego, ya que se le considera una especie pionera y de gran valor ecológico (Stecconi et al. 2000; Burns 1993), sobretodo en ambientes dominados por Austrocedrus chilensis (Don) Florin et Boutleje y N. dombeyi (Mirb.) Blume. En Chile el ñirre se distribuye entre los $36^{\circ} \mathrm{S}$ y $56^{\circ} \mathrm{S}$ y sus poblaciones cubren una superficie de aproximadamente 501.372 ha, que representan alrededor de $3,7 \%$ de la superficie total de bosque nativo (Navarro et al. 2008; Vidal \& Premoli 2004). Los bosques dominados por $N$. antarctica se concentran en la Región de Aysén (Rodríguez et al. 2008; Alvarado 2006). Según el Catastro de Bosque Nativo (CONAF-CONAMABIRF 1999) esta especie constituye parte de las coberturas vegetacionales Bosque achaparrado con ñirre como único componente y Matorral. Esta última cobertura, corresponde a un bosque mixto secundario generado principalmente por acción antrópica y donde el ñirre está presente junto a Embotrium coccineum J.R. Forst et G. Forst (notro), $N$. betuloides (Mirb.) Oerst. (coigue de Magallanes) y N. pumilio (Poepp. et Endl) y Krasser (lenga).

Trabajos que caracterizan la fauna de insectos asociada a Nothofagus en el sur de Chile, son aislados y se han realizado principalmente para componentes de la fauna humícola (Hammond et al. 2004; Covarrubias et al. 1992; Lanfranco 1977, 1980) y para insectos defoliadores de $N$. oblicua (Mirbel) Oersted (Lanfranco et al. 2001; Jerez 1996). En este aspecto, destacan los trabajos de McQuillan (1993), quien compara la fauna relacionada a Nothofagus, Lanfranco (1991) con insectos asociados a bosques de $N$. pumilio en Magallanes, Grandón (1996) quien analiza el estado fitosanitario de Nothofagus en Chile y Alvarado (2006) quien determina el estado sanitario de estos bosques en la zona de Coyhaique, categorizándolos en situación de decadencia. Los objetivos de este trabajo son caracterizar la fauna de insectos asociados al follaje en bosques puros de ñirre y matorral en la cuenca del río Baker, Región de Aysén, Chile y determinar si existen o no diferencias tanto estacionales como entre coberturas en la composición taxonómica de los gremios más representativos.

\section{MATERIALES Y MÉTODOS}

Área DE ESTUdio

La Región de Aysén del General Carlos Ibáñez del Campo

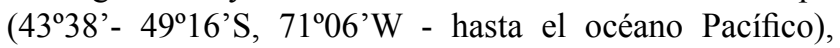
comprende una superficie de $106.990 \mathrm{Km}^{2}$ (Instituto Geográfico Militar 1982). El área de estudio se ubica en la cuenca del río Baker, donde están representados Bosques achaparrados de ñirre y Matorral con un $11,0 \%$ y $25,1 \%$ de cobertura, respectivamente. El Catastro de Bosque Nativo (CONAF-CONAMA-BIRF 1999) define como "Bosque Achaparrado" a formaciones boscosas donde el ñirre es el componente dominante con árboles de 2 a $8 \mathrm{~m}$ de altura y que están regidos por las condiciones ambientales imperantes en el lugar. Por el contrario, la cobertura "Matorral" corresponde a bosques mixtos secundarios generados por acción antrópica, con árboles menores a $2 \mathrm{~m}$ de altura y asociados principalmente a Embotrium coccineum (notro).

\section{Puntos DE MUeSTREO.}

Se efectuaron dos muestreos estacionales en octubre de 2006 (primavera) y enero de 2007 (verano), en sectores ubicados entre Puerto Bertrand y Fiordo Mitchell en la cuenca del río Baker $\left(47^{\circ} 2^{\prime} \mathrm{S}-72^{\circ} 48^{\prime} \mathrm{W}\right.$; $\left.48^{\circ} 01^{\prime}-73^{\circ} 10^{\prime} \mathrm{W}\right)$. Como unidades de estudio fueron seleccionados cinco bosques de tipo achaparrado y 10 de tipo matorral (Fig. 1), debido a la distinta representatividad de ambas coberturas en la cuenca. Adicionalmente, fueron incluidos muestreos en otras coberturas con presencia de ñirre, como es el caso de plantaciones forestales, estepas y otros tipos vegetacionales (Tabla 1).

\section{Método de Muestreo}

Los insectos fueron recolectados mediante sacudido de ramas con paraguas entomológico (Centella et al. 2003; Peña 1996; Saiz et al. 1989), técnica que permite la captura de estados larvarios o ninfales de insectos y adultos no voladores o bien con rangos de vuelo restringidos como es el caso de coleópteros, heterópteros y micro-himenópteros (Suckling et al. 1996). Aunque desde el punto de vista metodológico, este método es considerado de muestreo relativo ya que permite analizar poblaciones de insectos en un espacio o un tiempo, no permitiendo su correlación con unidades de área (densidad) como en el caso de métodos absolutos, es igualmente factible capturar un gran número de individuos (Southwood 1996). Para el análisis taxonómico, consideramos sólo las familias y/o especies propias de follaje (Peña 1996; Saíz et al. 1989). Asimismo, identificamos algunos estados inmaduros de insectos y estados de infestación de ramas por plantas y hongos parásitos (Alvarado 2006; Cerda \& Angulo 2000; Godoy et al. 2000; González \& Opazo 2000). La determinación de los taxa y su inclusión dentro de un determinado gremio fue corroborada mediante literatura especializada: Solervicens (2006), Hammond et al. (2004), Elgueta et al. (2002), Arias (2000), Morrone \& Coscarón (1998), Slipinski (1998), Peña (1996), Saiz et al. (1989), Bechyne (1973) y Jeannel (1962), entre otros, además de comparación con material del Museo de Zoología de la Universidad de Concepción (UCCC) y Museo Nacional de Historia Natural, Santiago (MNHN). La inserción de las especies en un gremio determinado fue estimada en base a caracteres morfológicos del aparato bucal y a aspectos ecológicos (alimentación y hábitat) conocidos a priori para los diferentes taxa. 


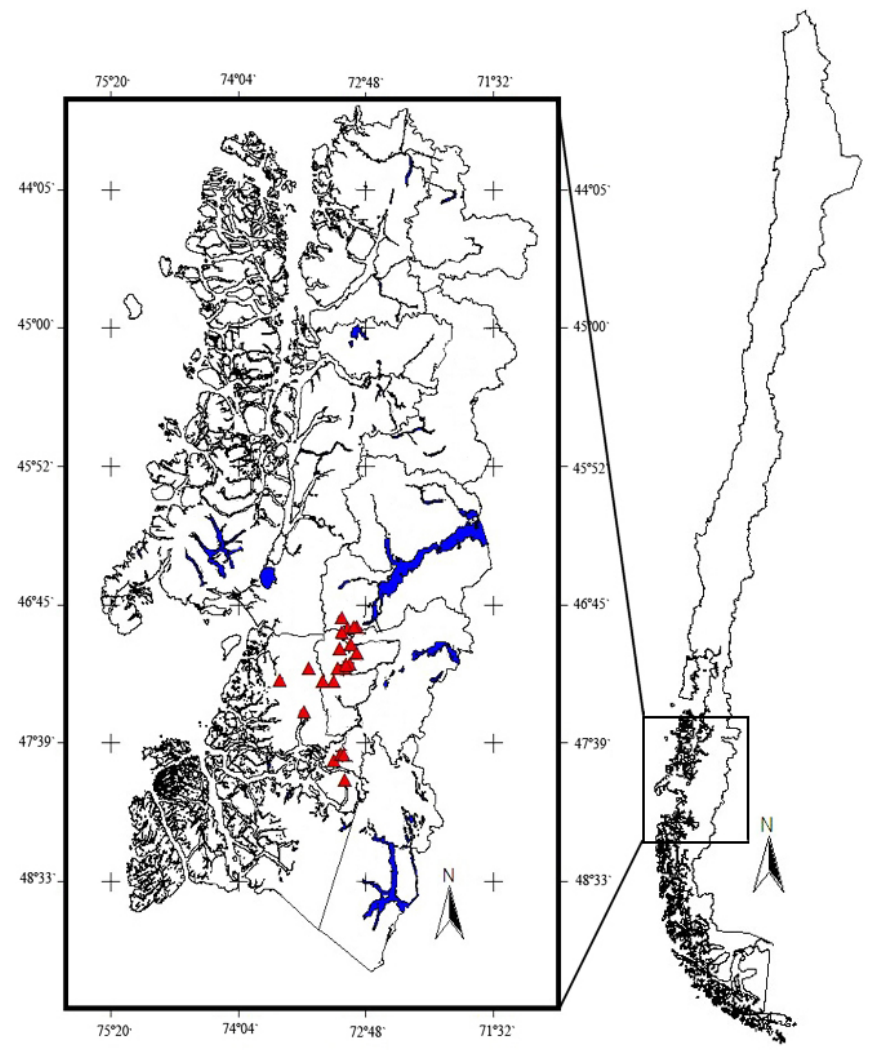

Figura 1: Mapa de los sitios de muestreo en la Región de Aysén, Chile, detallados en la Tabla 1.

FIgURE 1: Map and sampling points of Región de Aysen, Chile, from Table 1.

Tabla 1: Puntos de muestreo de insectos asociados a Nothofagus antarctica en la cuenca del río Baker al sur de Puerto Bertrand (47² $2^{\prime} \mathrm{S}$ - 7248`W), Región de Aysén, Chile. Se detalla las coordenadas (LS y LW) para verano y primavera.

TABLE 1: Sampling points of insects associated to Nothofagus antarctica at the Baker river basin to the south of Puerto Bertrand (47 $2^{\prime} \mathrm{S}$ - 7248` W), Región de Aysén, Chile. The coordinates (LS and LW) for summer and spring are provided.

\begin{tabular}{|c|c|c|c|c|c|}
\hline \multirow[t]{2}{*}{ Cobertura Vegetacional } & \multirow[t]{2}{*}{ Nombre sector } & \multicolumn{2}{|c|}{ Primavera } & \multicolumn{2}{|c|}{ Verano } \\
\hline & & $\mathbf{L S}$ & L O & LS S & LO \\
\hline \multirow[t]{5}{*}{ Bosque Achaparrado } & Predio SAG & $47^{\circ} 07^{\prime} 42^{\prime \prime}$ & $72^{\circ} 42^{\prime} 07^{\prime \prime}$ & $47^{\circ} 07^{\prime} 42^{\prime \prime}$ & $72^{\circ} 42^{\prime} 07^{\prime \prime}$ \\
\hline & El Manzano & $47^{\circ} 06^{\prime} 49^{\prime \prime}$ & $72^{\circ} 36^{\prime} 50^{\prime \prime}$ & $47^{\circ} 06^{\prime} 49^{\prime \prime}$ & $72^{\circ} 36^{\prime} 51^{\prime \prime}$ \\
\hline & Confluencia & & & $47^{\circ} 03^{\prime} 24^{\prime \prime}$ & $72^{\circ} 46^{\prime} 27^{\prime \prime}$ \\
\hline & La Colonia & $47^{\circ} 13^{\prime} 46^{\prime \prime}$ & $72^{\circ} 39^{\prime} 24^{\prime \prime}$ & $47^{\circ} 13^{\prime} 47^{\prime}$ & $72^{\circ} 39^{\prime} 23^{\prime \prime}$ \\
\hline & Barrancoso & $47^{\circ} 22^{\prime} 00^{\prime \prime}$ & $72^{\circ} 41^{\prime} 22^{\prime \prime}$ & $47^{\circ} 22^{\prime} 04^{\prime \prime}$ & $72^{\circ} 41^{\prime} 27^{\prime}$ \\
\hline \multirow[t]{9}{*}{ Matorral } & Los Maitenes & $47^{\circ} 09^{\prime} 19^{\prime \prime}$ & $72^{\circ} 45^{\prime} 47^{\prime}$ & $47^{\circ} 09^{`} 18^{\prime \prime}$ & $72^{\circ} 45^{\prime} 47^{\prime}$ \\
\hline & La Colonia & $47^{\circ} 15^{\prime} 54^{\prime \prime}$ & $72^{\circ} 45^{\prime} 41^{\prime \prime}$ & $47^{\circ} 15^{`} 54^{\prime \prime}$ & $72^{\circ} 45^{\prime} 41^{\prime \prime}$ \\
\hline & Los Ñadis & $47^{\circ} 29^{\prime} 01^{\prime \prime}$ & $72^{\circ} 54^{\prime} 18^{\prime \prime}$ & & \\
\hline & Barrancoso & $47^{\circ} 29^{\prime} 56^{\prime \prime}$ & $72^{\circ} 49^{\prime} 15^{\prime \prime}$ & & \\
\hline & Barrancoso & & & $47^{\circ} 28^{\prime} 33^{\prime}$ & $72^{\circ} 47^{\prime} 48^{\prime \prime}$ \\
\hline & Barrancoso & & & $47^{\circ} 23^{\prime} 21^{\prime \prime}$ & $72^{\circ} 42^{\prime} 13^{\prime \prime}$ \\
\hline & Los Nadis & & & $47^{\circ} 29^{\prime} 01^{\prime \prime}$ & $72^{\circ} 54^{\prime} 19^{\prime \prime}$ \\
\hline & Puerto Yungay & $47^{\circ} 55^{\prime} 40^{\prime \prime}$ & $73^{\circ} 20^{\prime} 05^{\prime \prime}$ & & \\
\hline & Pasarela Vargas & $47^{\circ} 41^{\prime} 55^{\prime}$ & $73^{\circ} 04^{\prime} 32^{\prime \prime}$ & & \\
\hline Plantación & Cochrane & $47^{\circ} 17^{\prime} 01^{\prime \prime}$ & $72^{\circ} 35^{\prime} 06^{\prime \prime}$ & $47^{\circ} 17^{\prime} 00^{\prime \prime}$ & $72^{\circ} 35^{\prime} 07^{\prime \prime}$ \\
\hline \multirow[t]{2}{*}{ Estepa } & El Manzano & $47^{\circ} 07^{\prime} 03^{\prime \prime}$ & $72^{\circ} 38^{\prime} 13^{\prime \prime}$ & & \\
\hline & Colonia & $47^{\circ} 17^{\prime} 07^{\prime \prime}$ & $72^{\circ} 35^{\prime} 39^{\prime \prime}$ & & \\
\hline \multirow[t]{3}{*}{ Otros } & Camino Fiordo Mitchell & $48^{\circ} 01^{\prime} 18^{\prime \prime}$ & $73^{\circ} 10^{\prime} 45^{\prime \prime}$ & & \\
\hline & Camino Fiordo Mitchell & $48^{\circ} 01^{\prime} 14^{\prime \prime}$ & $73^{\circ} 07^{\prime} 18^{\prime \prime}$ & & \\
\hline & Camino Fiordo Mitchell & $47^{\circ} 59^{\prime} 10^{\prime \prime}$ & $73^{\circ} 09^{\prime} 03^{\prime \prime}$ & & \\
\hline
\end{tabular}


COMPOSICIÓN TAXONÓMICA Y GREMIOS.

Se determinó la composición taxonómica de insectos adultos propios del follaje, insectos errantes y estados inmaduros, además de plantas y hongos parásitos presentes en las coberturas Bosque achaparrado y Matorral. Con el grupo taxonómico más representativo, analizamos la representatividad de sus distintos gremios, entendidos éstos como los grupos de especies que explotan un recurso común básico en forma semejante (Root 1967 sensu Krebs 1985) y clasificados según literatura especializada detallada anteriormente. La abundancia relativa de cada gremio fue analizada para ambas coberturas vegetacionales (Bosque achaparrado y Matorral) y para ambas estaciones de muestreo (primavera y verano).

ANÁLISIS DE DATOS

Se calculó la diversidad de la cuenca del río Baker, medida por la abundancia (total y promedio), riqueza y frecuencia total de las especies, así como de los distintos gremios, para ambas coberturas vegetacionales (Bosque achaparrado y Matorral) y para ambas estaciones de muestreo (primavera $\mathrm{y}$ verano). Las abundancias promedio fueron comparadas mediante la prueba de hipótesis ANOVA en el programa PAST 1,82b (Hammer et al. 2001) para el total de las especies y para los gremios del orden de insectos más representativo.

\section{RESULTADOS}

COMPOSICIÓN TAXONÓMICA

En el follaje de ñirre fueron registrados seis órdenes de insectos, de los cuales el más representativo fue Coleoptera con 10 familias y 37 especies, seguido por Heteroptera (Hemiptera) con cinco familias y ocho especies (Tabla 2, Fig. 2). Los taxa errantes para el follaje estuvieron representados por insectos depredadores y/o voladores, entre ellos coleópteros de las familias Staphylinidae (Atheta sp.), Carabidae (Euproctinus fasciatus (Solier), Mimodromius cyanipennis (Brullé)) y Scirtidae (Cyphon sp.). También fueron registrados himenópteros de la familia Formicidae (Camponotus chilensis (Spinola)), plecópteros de la familia Grypopterygidae y dípteros de las familias Tabanidae, Muscidae y Tipulidae.

Entre los coleópteros, las familias más representativas fueron Curculionidae con 14 géneros y 19 especies, y Chrysomelidae con seis géneros y seis especies. Las especies Polydrusus nothofagi Kuschel (n=28), Polydrusus roseus Bl. $(\mathrm{n}=25)$ (Curculionidae), Ericmodes tarsalis Slipinski $(\mathrm{n}=20)$ (Protocucujidae) y Polychisme $\mathrm{sp}(\mathrm{n}=20)$ (Heteroptera), presentaron las mayores abundancias en ambas coberturas vegetacionales y en ambos periodos de muestreo. Las especies constantes para el total de los muestreos $(\mathrm{S}$ total $=18)$ fueron $P$. nothofagi $(\mathrm{S}=9 / 18), P$. roseus $(\mathrm{S}=9 / 18)$, (Curculionidae), Polychisme sp. $(\mathrm{S}=8 / 18)$, Ditomotarsus punctiventris Spin. ( $\mathrm{S}=7 / 18)$, (Heteroptera), Adalia deficiens Mulsant $(\mathrm{S}=7 / 18)$ (Coccinellidae), y Eurymetopum eburneocinctum (Spin.) ( $\mathrm{S}=7 / 18)$ (Cleridae). No se registraron diferencias significativas en el promedio de abundancias de los insectos encontrados para Bosque achaparrado y Matorral en los periodos de muestreo $(\mathrm{F}=$ $1,094 ; \mathrm{p}=0,3525)$.

Con respecto a los estados inmaduros de insectos se registró la presencia de larvas de Lepidoptera (Geometridae y Noctuidae), ninfas de Lygaeidae y Pentatomidae (Heteroptera) y Psocoptera.

En relación a la presencia de plantas parásitas, algunos ñirres presentaron infestación por Misodendrum quadriflorum G. Don, planta hemi-parásita (Rodríguez et al. 2008) y Cyttaria harioti Fisco, hongo ascomicetes inductor de hipertrofias o tumores en ramillas, ramas o fuste joven sobre los que fructifica. Además, se registró la presencia de cecidias inducidas por avispas de la familia Cynipidae, en cuyo interior oviposita Noterapion meorrhynchum (Phil. \& Phil.), curculiónido habitante de la cavidad agallícola (Kissinger 2002). Finalmente, encontramos en abundancia larvas de Ormiscodes sp. (Lepidoptera: Saturnidae), principalmente en terrenos poco drenados.

\section{GREMIOS}

El gremio más representativo corresponde al de tipo fitófago, representado para cada cobertura vegetacional en más de un 50\% del total de gremios (Fig. 2). Con respecto a la abundancia de depredadores, ésta fue mayor en primavera (Bosque achaparrado 35\% y Matorral 34\%) que en verano (Bosque achaparrado $7,9 \%$ y Matorral 9,8\%). La presencia de xilófagos fue constatada en Matorral en verano. Por otra parte, el gremio de coleópteros omnívoros presentó poca representatividad $(<20 \%$ ) (Fig. 2). No se registraron diferencias significativas para la abundancia promedio de los gremios en los diferentes periodos de muestreo ( $\mathrm{F}$ $=0,3628 ; \mathrm{p}=0,781)$. 


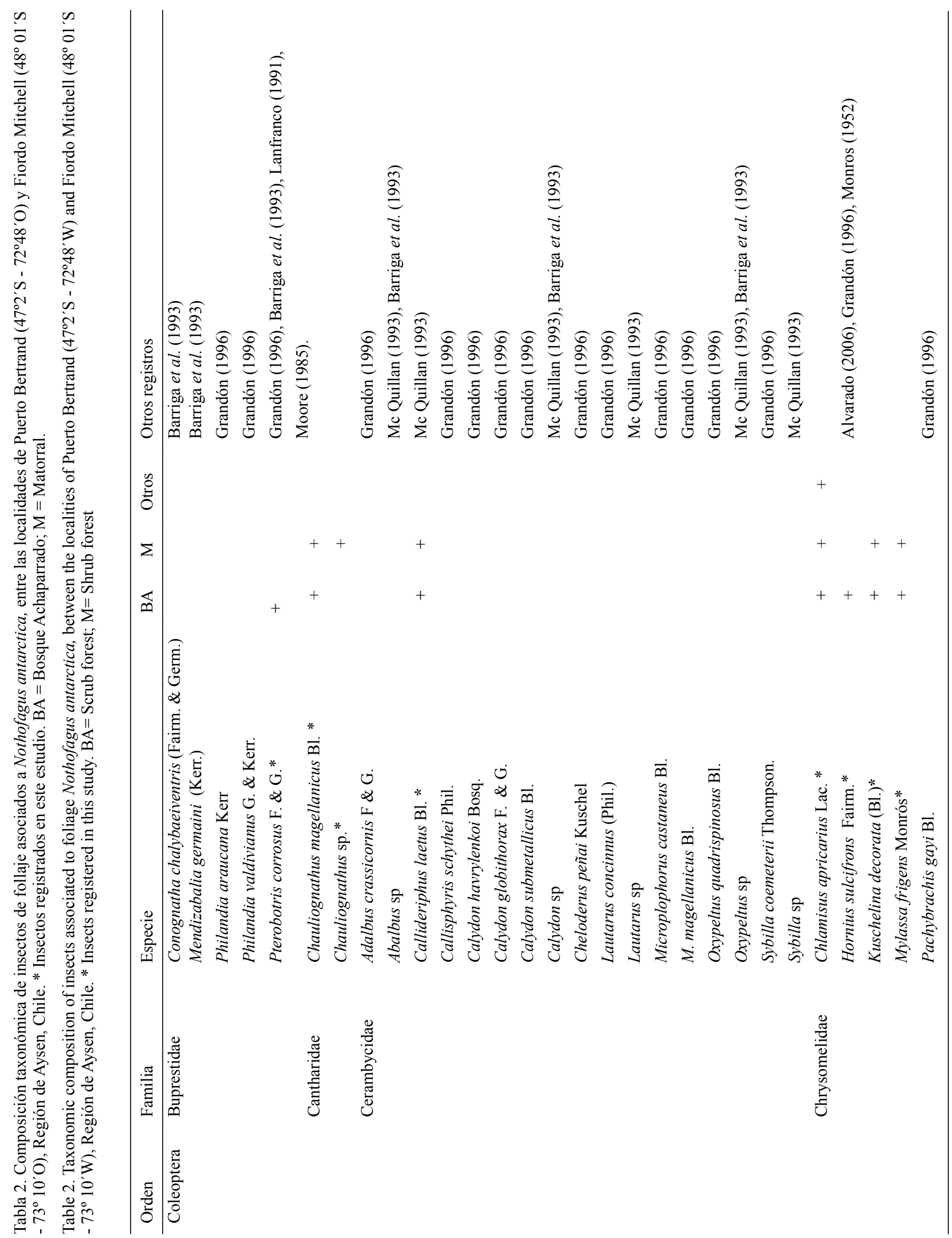




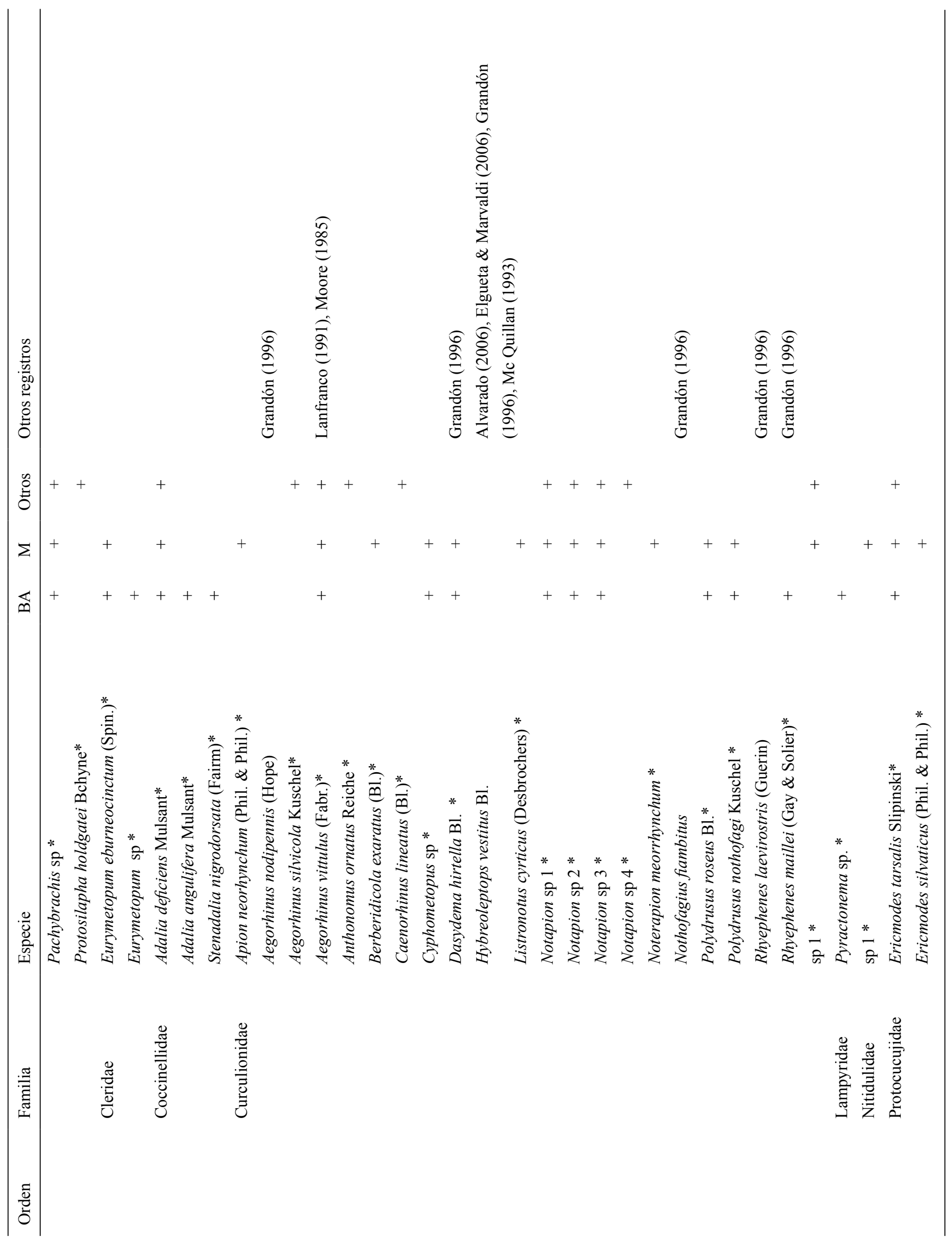




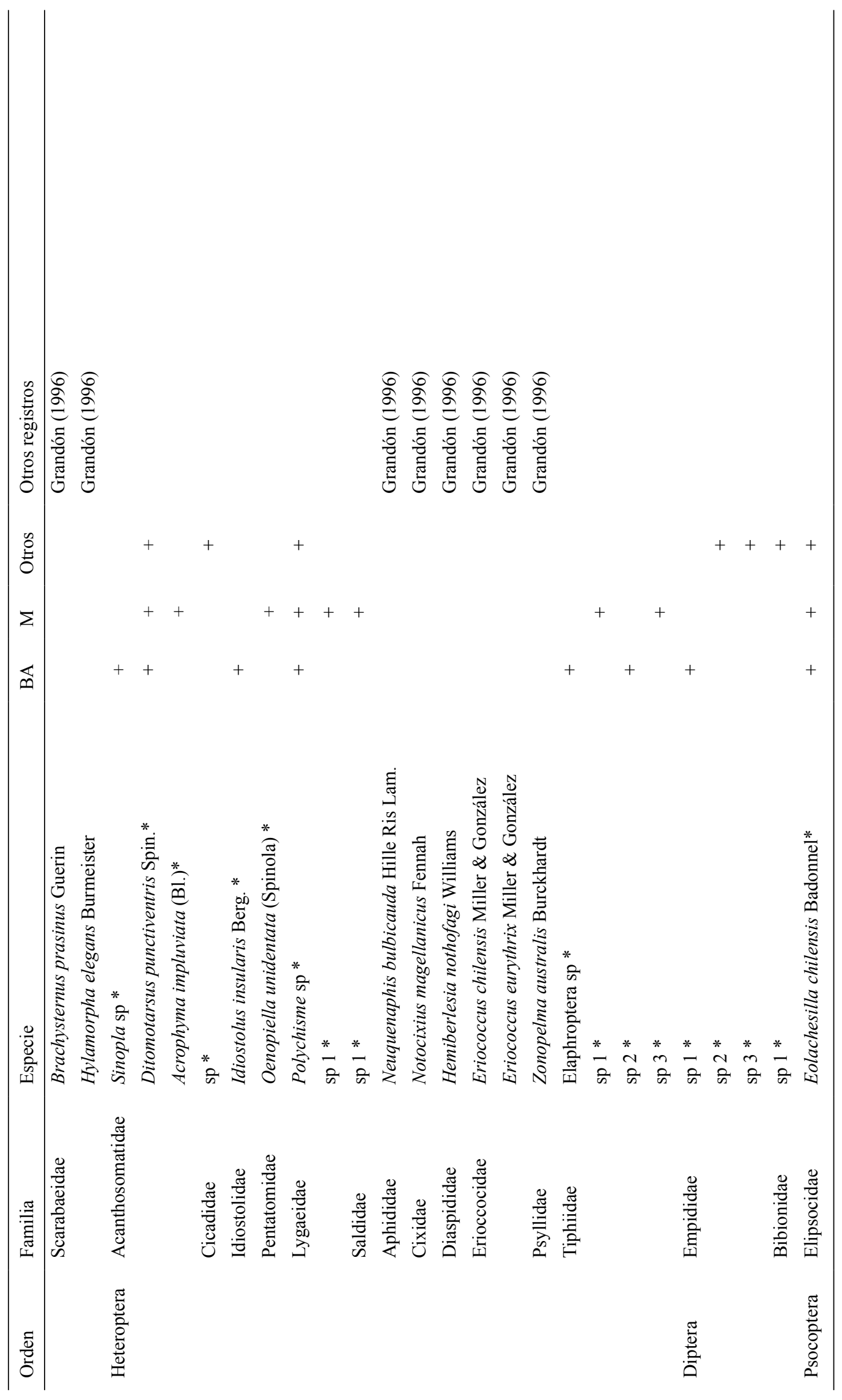




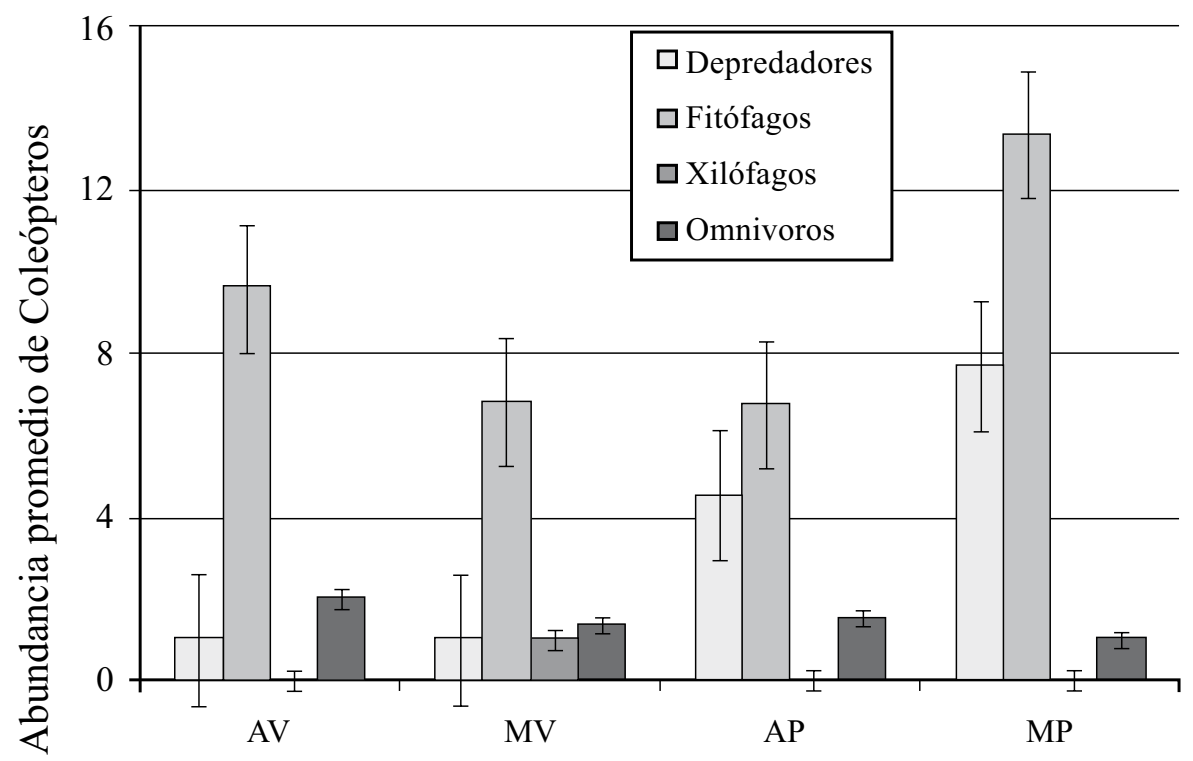

FiguRa 2. Abundancia promedio de coleópteros para cada gremio, cobertura vegetacional y estación climática colectados entre las localidades

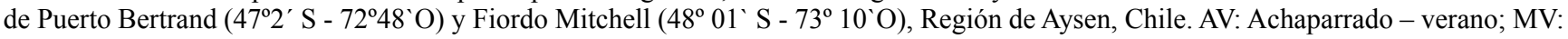
Matorral - verano; AP: Achaparrado Primavera y MP: Matorral Primavera. Se muestra la desviación estándar para cada gremio.

FigURE 2. Mean of abundance for beetles for each guilds, forest cover and calendar season collected between the localities of Puerto Bertrand ( $\left.47^{\circ} 2^{\prime} \mathrm{S}-72^{\circ} 48^{\prime} \mathrm{W}\right)$ and Fiordo Mitchell (48 $\left.01^{\circ} \mathrm{S}-73^{\circ} 10^{\prime} \mathrm{W}\right)$, Región de Aysen, Chile. AV: Scrub forest - summer; MV: Shrub forest - summer; AP: Scrub forest - spring and MP: shrub forest- spring. Standard deviation for each guild is showed.

\section{DISCUSIÓN}

En la zona sur de la Región de Aysén, Chile, registramos seis órdenes de insectos asociados al follaje de $N$. antarctica, siendo Coleoptera el más representativo y Curculionidae la familia con mayor riqueza; esta familia presenta una gran diversidad morfológica y biológica y sus especies exclusivamente de hábitos fitófagos, están presentes mayoritariamente en ambientes áridos y semiáridos (Elgueta \& Marvaldi 2006), como ocurre en la cuenca del río Baker.

Mc Quillan (1993), Barriga et al. (1993) y Grandón (1996) registraron en ñirre especies de Cerambycidae, Abalbus sp., Calydon sp., Oxypeltus sp., Lautarus sp. y Sibylla sp. y una de Curculionidae, Hybreoleptops sp., no encontradas en este muestreo. Aunque Alvarado (2006), cita a Hybreoleptops vestitus Bl. (Curculionidae) en localidades cercanas a Coyhaique, para Elgueta \& Marvaldi (2006) el limite sur de esta especie en Chile correspondería a Valdivia.

Por otra parte, Barriga et al. (1993) indican la presencia en ñirre de los bupréstidos Conognatha chalybaeiventris (F. \& G.) y Mendizabalia germaini (Kerremans), además de Pterobothris corrosus F. \& G., especie típica de zonas altas de montaña (Peña 1996) y registrada en este estudio. Grandón (1996), por su parte, identifica también a Philandia araucana Kerr. y P. valdivianus G \& Kerr como habitantes del ñirre. Alvarado (2006) además, indica la presencia del crisomélido Hornius sulcifrons $\mathrm{F}$. en los alrededores de Coyhaique. Al respecto, Hornius es un género endémico de la región subantártica y está asociado a bosques de Nothofagus (Monros 1952); de sus dos especies, H. sulcifrons es la más austral y se asocia trófica y reproductivamente a ñirre (Jerez 1996). En nuestro estudio, encontramos oviposturas de $H$. sulcifrons en los vértices de las ramas de ñirre en primavera. Observaciones personales, han determinado la presencia en otoño de adultos de esta especie, en bosques achaparrados de ñirre presentes en sectores aledaños al río Baker, al norte de Cochrane.

Adicionalmente, Grandón (1996) registra la presencia en ñirre de especies de hábitos succionadores, los heterópteros (homópteros) de las familias Aphididae, Cixidae, Diaspididae, Erioccocidae y Psyllidae, no encontrados en este estudio.

Con respecto al estado fitosanitario, Grandón (1996) sostiene que en Chile $N$. antarctica se encuentra en un estado sanitario 
regular debido a que sus insectos defoliadores, agallíferos y succionadores producen una pérdida de vigor en el árbol. Más específicamente, Alvarado (2006) concluye que los bosques de ñirre de la Región de Aysén se encuentran en estado de decadencia debido a la gran cantidad de defoliación a la que están expuestos, lo que los hace susceptibles a ser atacados por algún agente de daño durante la siguiente temporada. Dentro de los patógenos más evidentes para ñirre, encontramos a Misodendrum quadriflorum D.C. y Cyttaria harioti Fisch (hongo ascomicete). González \& Opazo (2000) y Alvarado (2006), citan, además, a C. darwini Berk y $C$. hookeri Berk como hongos inductores de tumores para ñirre. Por otra parte, el mayor daño evidenciado en ambientes secos, fue la abundante defoliación producida por larvas de Ormiscodes sp. (Lepidoptera: Saturnidae). Estas larvas pueden corresponder a $O$. amphinome que por distribución estaría registrada para la zona (Cerda \& Angulo 2000; Veblen et al. 1995). Sin embargo, estudios realizados en esta misma zona y mediante trampas de luz registraron adultos de $O$. joiceyi y $O$. cognata en la temporada anterior (Ortiz 2007).

Algunos de los insectos considerados dañinos para los bosques en general y encontrados en ñirre son: Pterobothris corrosus F. \& G. (Buprestidae) y Aegorhinus vitulus (Fabr.) (Curculionidae); ambas especies pueden ser encontradas también en $N$. pumilio según lo documentado por Moore (1985) y Lanfranco (1991). Además, constatamos la presencia de Rhyephenes maillei (Gay \& Solier) (Curculionidae), quien consume los brotes foliares del ñirre y su larva xilófaga taladra la madera (sensu Cerda $\&$ Angulo 2000).

Los coleópteros $P$. nothofagi, $P$. roseus, D. punctiventris, $A$. deficiens y E. eburneocinctum además del Lygaeidae Polychisme sp., fueron las especies más representativas de los muestreos, siendo definidas como especies constantes para el ñirre.

En cuanto a la presencia de diversos gremios de insectos, el fitófago fue el más representativo en los insectos de follaje, en las estaciones de primavera y verano tanto en bosques achaparrados como matorral. En el caso de los depredadores, éstos fueron más abundantes en primavera, posiblemente por la mayor cantidad de estados inmaduros de insectos en el follaje y que les pueden servir de alimento. Las abundancias de omnívoros fueron menores y sólo en un ambiente fueron registrados xilófagos (matorral). Esto debe ser evaluado en detalle y concluido con mesura, tomando en cuenta los meses en que fueron realizados estos muestreos, en un año con Efecto niño - Oscilación del Sur.

Debido al gran estrés ambiental diario y anual de la Región de Aysén, situación evidenciada también en la Región de Magallanes (Lanfranco 1991, 1980, 1977), los ciclos biológicos de los insectos pueden ser muy rápidos o estar desfasados fenológicamente y la vagilidad de los individuos (y por ende su captura), puede variar diariamente y más aún estacionalmente. Un aumento del estrés del suelo con respecto al agua y a los nutrientes, puede reducir la abundancia de herbívoros y por consiguiente de depredadores y parasitoides en insectos de follaje. Por ello, estimamos conveniente realizar un estudio anual de la diversidad de estos insectos y combinar esta metodología con otras técnicas de muestreo, como por ejemplo, la utilización de trampas de luz y muestreos de suelo, para abarcar posibles cambios en la composición de especies a lo largo de las estaciones del año de acuerdo a la fenología de la planta y a su sincronización con los ciclos de vida de los insectos.

\section{AGRADECIMIENTOS}

A Mario Elgueta del Museo Nacional de Historia Natural (MNHN) por la disponibilidad para la revisión del material y la ayuda en la identificación de insectos. A Gerardo Arriagada por la apoyo en la identificación del material. A Raúl Briones y Jonathan Guzmán por la ayuda en el muestreo. A Ariel Farías, Dolly Lanfranco y Sergio Estay por la revisión de este manuscrito. Finalmente a Lafayette Eaton por su ayuda en la revisión del abstract. Este estudio fue financiado por el Proyecto DIUC No 206.113.72-3 de la Dirección de Investigación, Universidad de Concepción.

\section{BIBLIOGRAFÍA}

Alvarado, A. 2006. Determinación del estado sanitario de los bosques de ñirre (Nothofagus antarctica (G. Forster) Oersted) en la XI Región. Proyecto de Título, Departamento de Ciencias Forestales, Facultad de Agronomía e Ingeniería Forestal, Pontificia Universidad Católica de Chile. 137 pp.

Arias, E. 2000. Coleópteros de Chile. Litografía Valente. Santiago de Chile. 209 pp.

Barriga, J., Curkovic, T., Fichet, T., Henríquez, J. \& Magaya, J. 1993. Nuevos antecedentes de Coleópteros xilófagos y plantas hospederas en Chile, con una recopilación de citas previas. Revista Chilena de Entomología 20:65-91.

Bechyne, J. 1973. Notas sobre algunos phytophaga de origen paleantartico. Revista Chilena de Entomología 7:25-30.

BuRns, B. 1993. Fire-induced dynamics of Araucaria araucana - Nothofagus antarctica forest in the southern Andes. Journal of Biogeography 20:669-685.

Centella, C., Jerez, V., Gonzalez, U. \& Bittner, M. 2003. Especialización en el uso de hospederos de Dictyneis asperatus (Blanchard, 1851) en un fragmento de vegetación esclerófila - higrófila en la península de Hualpén, Chile. Revista Chilena de Historia Natural 76:391-400.

Cerda, L. \& Angulo. A. 2000. Insectos asociados a bosques del centro sur de Chile. En: Agentes de daño en el bosque nativo (Ed. Baldini \& Pancel), pp. 201-269. Editorial Universitaria. 
Coley, P. 1987. Patrones en las defensas de las plantas: ¿Por qué los herbívoros prefieren ciertas plantas?. Revista de Biología Tropical 35(supl. 1):151-164.

Conaf-Conama-Birf. 1999. Catastro y evaluación de los recursos vegetacionales nativos de Chile. Informe Regional Undécima Región.

Covarrubias, R., Covarrubias, C. \& Mellado. I. 1992. Microartrópodos en suelos de bosques de Nothofagus pumilio en parques nacionales de Chile. Acta Entomológica Chilena 17:195-210.

Elgueta, M., Mondaca, J. \& Vera, A. 2002. Fauna de coleópteros (Insecta: Coleoptera) del Parque Nacional San Rafael, Aysén - Chile. Boletín del Museo Nacional de Historia Natural. Chile 51:103-115.

Elgueta, M., \& Marvaldi, A. 2006. Lista sistemática de las especies de Curculionoidea (Insecta: Coleóptera) presentes en Chile, con su sinonimia. Boletín del Museo Nacional de Historia Natural, Chile 55:113-153.

Godoy, R., Ramírez, C. \& Puentes, O. 2000. Plantas parásitas vasculares de Chile. En: Agentes de daño en el bosque nativo (Ed. Baldini \& Pancel), pp: 53-86. Primera edición, Editorial Universitaria. 408 pp.

GonzÁlez, G. \& Opazo, A. 2000. Enfermedades fungosas y otras. En: Agentes de daño en el bosque nativo (Ed. Baldini \& Pancel), pp: 89-135. Primera edición, Ed. Universitaria. $408 \mathrm{pp}$.

Grandón, F. 1996. Análisis fitosanitario de los Nothofagus de Chile, desde el punto de vista entomológico. Tesis de Grado para optar al Título de Ingeniero Forestal, Facultad de Ciencias forestales, Universidad Austral de Chile. 92 pp.

Hammer Q, Herper, D.A.T. \& Ryan, P.D. 2001. PAST: Palaeontological Statistics software package for education and data analysis. Palaeontologia Electronica 4(1):9 pp.

Hammond, P., Jackson, K., Hine S. \& Deboise, E. 2004. Competition and origin of forest beetles (Insecta: Coleoptera) assemblages in Region XI, Chile. The Natural History Museum, London. 294 pp.

Instituto Geográfico Militar. 1982. Atlas de la República de Chile. Segunda Edición Impresora TTGG, Instituto Geográfico Militar, Chile. 350 pp.

JAKSIC, F. 2001. Ecología de comunidades. Primera edición, Ed. Universidad Católica de Chile. 233 pp.

Jeannel, R. 1962. Les Silphidae, Leiodidae, Camiaridae et Catopidae de Paleantarctide occidentale. En: Biologie de L'Amérique Australe. Volumen I. (Ed. Delamare \& Rapoport) Editions du Centre National de la Recherche Scientifique France et Consejo National de Investigaciones Científicas y Técnicas, Francia.

Jerez, V. 1996. Biology and phylogenetic remarks of the subantarctic genera Hornius, Stenomela and Dictyneis (Chrysomelidae: Eumolpinae). En: Chrysomelidae Biology (Ed, Jolivet \& Cox), pp: 239 - 258. General Studies vol 3. SPB Academic Publishing Amsterdam, The Netherlands.

Jerez, V. 2006. Patrones de diversidad de Chrysomelidae (InsectaColeoptera) en la Cordillera de la Costa de Chile central y sur. En: Historia, biodiversidad y ecología de los bosques costeros de Chile (Ed. Smith-Ramírez, Armesto \& Valdovinos), pp: 340-350. Primera edición, Editorial Universitaria, Chile.

KISSINGER, DG. 2002. Review of Noterapion Kissinger from Chile and Argentina (Coleoptera: Apionidae). Insecta Mundi 16(4):220-246.

KREBS, CH. 1985. Ecología. Estudio de la Distribución y la Abundancia. Segunda edición. Editorial Harla. México. $751 \mathrm{pp}$.
LANFranco, D. 1977. Entomofauna asociada a los bosques de Nothofagus pumilio (POEPP et ENDL.) Krasser en la Región de Magallanes. Anales del Instituto de Patagonia, Chile 8:319-348.

LANFrAnCo, D. 1980. Estudios entomológicos en el Archipiélago del Cabo de Hornos. Anales del Instituto de la Patagonia, Chile 11:281-291.

LANFranco, D. 1991. Sinopsis de los insectos que atacan bosques de Lenga (Nothofagus pumilio (Poepp. Et Ende.)) Krass. en Magallanes. Anales del Instituto de la Patagonia, serie Ciencias Naturales, Chile 20(1):89-93.

Lanfranco, D., Rojas, E., Rios, R. \& Ruiz C. 2001. Insect defoliators of Nothofagus oblicua (Roble) in South Chile: Two Years Monitoring Species and Their Damage. En: Proceedings: integrated management and dynamics of forest defoliating insects (Eds Liebhold, McManus, Otvos \& Fosbroke), pp: 91-103.U.S. Department of Agriculture, Forest Service, Northeastern Research Station.

Mc Quillan, P. 1993. Nothofagus (Fagaceae) and its invertebrate fauna-and overview and prelimininary síntesis. Biological Journal of the Linnean Society 49:317-354.

Medel, R., Botto-Maham, C., Smith-Ramírez, C., Méndez, M., Ossa, C., CAputo, L. \& GonzÁlez., W. 2002. Historia natural cuantitativa de la relación parásito-hospedero: el sistema Tristerix- cactáceas en Chile semiárido. Revista Chilena de Historia Natural 75:127-140.

Monros, W. F. 1952. Notas sobre algunas Eumolpinae neotropicales. Revista Chilena de Entomología 2:187-196.

Moore, T. 1985. Aporte al conocimiento de los Bupréstidos de Chile (Coleóptera: Buprestidae) Segunda nota. Revista Chilena de Entomología 12:113-139.

Morrone, J.J. \& Coscarón S. (eds.). 1998. Biodiversidad de Artrópodos Argentinos, una perspectiva biotaxonómica. Primera edición, Ed. del sur, Argentina. 599 pp.

Navarro, R., Rosenfeld, M., Pérez-aranda, J., Padrón, E., Guzmán, J.R., Hernández R. \& GonzÁlez. L. 2008. Evaluación de la mortalidad de bosques de ñirre (Nothofagus antarctica) en la Patagonia chilena mediante imágenes Landsat TM y ETM+ BOSQUE 29(1):65-73.

Ortiz, J.C. 2007. Proyecto Hidroeléctrico Hidroaysén. Determinación de la línea de base de fauna terrestre en la zona sur de la región de Aysén. Resumen Ejecutivo.80 pp. http://www.hidroaysen.cl/site/PDF/Documentos/Res Ejecutivo_Fauna_Terrestre.pdf

PeÑA, L. 1996. Introducción al estudio de los insectos de Chile. Cuarta edición. Ed. Universitaria, Chile. 253 pp.

Rodríguez, R., Marticorena, A . \& Teneb, E. 2008. Plantas vasculares de los ríos Baker y Pascua, Región de Aysén, Chile. Gayana Botánica 65(1):39-70.

Rousseaux, M.C., Julkenen-Titto, R., Searles, P.S., Scopel, A.L., Aphalo, P.J. \& Ballaré C.L. 2004. Solar UV-B radiation affects leaf quality and insect herbivory in the southern beech tree Nothofagus antarctica. Oecologia 138:505-512

Saíz, F., Solervicens, J. \&. OJedA, P. 1989. Coleópteros del Parque Nacional La Campana y Chile central. Editorial Universidad de Valparaíso, Chile. 124 pp

SLIPINSKI, S.A. 1998. Revision and phylogeny of Protocucujidae (Coleoptera, Cucujoidea). Annales Zoologici 48: 273-296.

Solervicens, J. 2006. Diversidad y endemismos de Cleridae (Coleoptera) del Parque Nacional Nahuelbuta, VIII Región, Chile. En: Historia, biodiversidad y ecología de los bosques costeros de Chile (Eds. Smith-Ramírez, Armesto \& Valdovinos), pp: 324-334. Primera edición, Editorial Universitaria, Chile.

SouthwooD, T.R.E. 1996. Ecological methods, with particular 
reference to the study of insect populations. Second edition, Chapman \& Hall. 524 pp.

Stecconi, M., Puntieri, J. \& Barthélémy, D. 2000. Annual shootgrowth in Nothofagus antarctica (G. Foster) Oersted (Nothofagaceae) from northern Patagonia. Trees 14:289296.

Sucking, D.M, Burnip, G.M., GibB, A.R., Stavely, F.J.L. \& Wratten, S.D. 1996. Comparison of suction an beating tray sampling for apple pests ad their natural enemies. Proceedings of the $49^{\text {th }}$ New Zealand Plant Protection Conference: $41-47$.
Veblen, T., Kitzberger, T., Burns, B. \& Rebertus, A. 1995. Perturbaciones y dinámica de regeneración en bosques andinos del sur de Chile y Argentina. En: Ecología de los bosques nativos de Chile (Eds. Armesto, Villagrán \& Arroyo), pp: 169-197. Primera edición, Ed. Universitaria.

Vidal, R. \& Premoli, A.2004. Variación en Nothofagus antarctica (Foster) Oerst. (Ñirre Nire). En: Variación Intraespecífica en las especies arbóreas de los bosques templados de Chile y Argentina (Eds. Donoso, Premli,Gallo \& Ipinza), pp: 173 - 185. Primera edición, Ed. Universitaria.

Recibido: 25.05 .10

Aceptado: 29.09 .10 